\title{
Respiração lenta e profunda aumenta a modulação vagal em gestantes
}

\author{
Deep and slow breathing increases vagal modulation in pregnant women
}

\author{
Ana Claudia Silva Farche ${ }^{1} \bowtie$, Soraia Pilon Jürgensen ${ }^{1}$, Paulo Giusti Rossi ${ }^{1}$, \\ Anielle Cristhine de Medeiros Takahashi', Audrey Borghi-Silva ${ }^{1}$
}

${ }^{1}$ Universidade Federal de São Carlos (UFSCar), Departamento de Fisioterapia. São Carlos, SP.

\section{RESUMO}

OBJETIVOS: Comparar a resposta da modulação autonômica da frequência cardíaca de gestantes e mulheres não gestantes, antes e após a manobra de acentuação da arritmia sinusal respiratória.

MÉTODOS: Foi realizado um estudo transversal com amostra de 24 mulheres, divididas em dois grupos: grupo gestantes (independente da idade gestacional) e grupo controle (não gestantes). A avaliação consistiu em registro dos intervalos RR por um cardiofrequencímetro, para análise de índices lineares e não lineares da variabilidade da frequência cardíaca. As avaliações foram feitas no repouso em decúbito lateral esquerdo, antes e após a manobra de acentuação da arritmia sinusal respiratória, que consiste em respirações lentas de cinco a seis ciclos por minuto. A razão expiração/inspiração e a diferença entre a inspiração e a expiração foram avaliadas durante a manobra. Foram comparados os resultados obtidos entre os dois grupos (gestantes e não gestantes), assim como os obtidos em cada grupo, antes e após a manobra. Para a análise estatística o nível de significância considerado foi $\mathrm{p}<0,05$.

RESULTADOS: Os índices lineares mostraram valores iniciais da modulação simpática (banda de baixa frequência) aumentados nas gestantes quando comparadas às mulheres não gestantes (gestantes: $68,9 \pm 28,1$; controles: $49,3 \pm 11,7 ; \mathrm{p}=0,002$ ). Após a manobra respiratória, $\mathrm{o}$ índice obtido pela raiz quadrada da média do quadrado das diferenças entre os intervalos RR normais adjacentes aumentou no grupo gestantes (pré- manobra: $34,5 \pm 5,7$; pós-manobra: $38,9 \pm 5,8 ; \mathrm{p}=0,027$ ), indicando aumento da atuação parassimpática para essa população após a aplicação da manobra. Além disso, houve aumento dos valores de complexidade, sugeridos pela entropia de Shanon após a manobra, para ambos os grupos, sendo o aumento maior nas gestantes. Em relação aos índices avaliados durante a manobra, ambos os grupos apresentaram valores da razão expiração/inspiração maiores que 1, o que sugere integridade do sistema nervoso autônomo.

CONCLUSÕES: Houve melhora da regulação do sistema nervoso autônomo ao final da manobra respiratória para as gestantes, sugerindo que o padrão de respiração lenta e profunda possa aumentar a modulação vagal e conferir efeito cardioprotetor, além de ocasionar relaxamento e sensação de bem-estar.

DESCRITORES: gravidez; respiração; sistema cardiovascular; frequência cardíaca; arritmia sinusal respiratória; sistema nervoso autônomo.

\section{ABSTRACT}

AIMS: To compare the responses of the heart rate autonomic modulation in pregnant and non-pregnant women before and after the respiratory sinus arrhythmia maneuver.

METHODS: A cross-sectional study was conducted with a sample of 24 women, divided into two groups: pregnant group (independent of gestational age) and control group (non-pregnant). The evaluation consisted of recording the RR intervals using a cardiofrequencimeter, for analysis of linear and nonlinear indices of heart rate variability. The evaluations were made at rest in left lateral decubitus, before and after the maneuver of accentuation of respiratory sinus arrhythmia, consisting of slow breaths five to six cycles per minute. The expiration/inspiration ratio and the difference between inspiration and expiration were evaluated during the maneuver. Results were compared between the two groups (pregnant and non-pregnant), as well as those obtained in each group before and after the maneuver. For the statistical analysis the level of significance considered was $\mathrm{p}<0.05$.

RESULTS: Linear indices showed increased baseline sympathetic modulation (low frequency band) in pregnant women when compared to non-pregnant women (pregnant women: $68.9 \pm 28.1$; non pregnant women: $49.3 \pm 11.7 ; \mathrm{p}=0,002$ ). After the respiratory maneuver, the root mean square of successive differences in the RR intervals index increased in the pregnant group (pre-maneuver: $34.5 \pm 5.7$; post-maneuver: 38.9 \pm 5.8 ; $\mathrm{p}=0,002)$ indicating an increase in the parasympathetic system for this population after the maneuver. In addition, there was an increase in the complexity values after the maneuver, suggested by the Shanon entropy for both groups, being the increase higher in the pregnant women. Regarding the indices evaluated during the respiratory sinus arrhythmia maneuver, both groups presented expiration/inspiration ratio values greater than 1, which suggests integrity of the autonomic nervous system.

CONCLUSIONS: There was an improvement in the regulation of the autonomic nervous system after the respiratory maneuver for pregnant women, suggesting that the slow and deep breathing pattern may increase vagal modulation and confer a cardioprotective effect, besides causing relaxation and a sense of well-being.

KEY WORDS: pregnancy; respiration; cardiovascular system; heart rate; respiratory sinus arrhythmia; autonomic nervous system. 
Abreviaturas: AF, banda de alta frequência; $\mathrm{BF}$, banda de baixa frequência; $\mathrm{BF} / \mathrm{AF}$, razão entre áreas absolutas de baixa e alta frequência; E/I, razão da expiração pela inspiração; FC, frequência cardíaca; FR, frequência respiratória; GG, grupo de gestantes; $\mathrm{GC}$, grupo de mulheres não gestantes; $\Delta \mathrm{IE}$, diferença entre a inspiração e a expiração; IMC, índice de massa corporal; iRR, intervalos RR; mASR, manobra de acentuação da arritmia sinusal respiratória; PA, pressão arterial; RMSSD, raiz quadrada da média do quadrado das diferenças entre os intervalos RR normais adjacentes; VFC, variabilidade da frequência cardíaca.

\section{INTRODUÇÃO}

Durante a gestação, a volemia sanguínea, o débito cardíaco e o volume sistólico aumentam, o que leva a uma sobrecarga cronotrópica para manter a homeostase [1-3]. Assim, para suprir a demanda de aporte sanguíneo para o feto, o sistema nervoso autônomo atua elevando a frequência cardíaca (FC). Esta capacidade de adaptação a diferentes estímulos é resultado da atuação adequada do sistema nervoso autônomo [4].

Desta forma, o estudo da modulação autonômica cardíaca por meio da variabilidade da frequência cardíaca (VFC) torna-se uma ferramenta importante para avaliar a integridade deste sistema [1, 4-6]. Além da análise da VFC, um teste autonômico de grande importância é a manobra de acentuação da arritmia sinusal respiratória (mASR). Essa manobra consiste em respirações lentas de cinco a seis ciclos por minuto [7] e tem sido utilizada em diferentes populações para controle da dor e relaxamento muscular devido à interação entre as vias autonômicas e o núcleo do trato solitário [8].

O núcleo do trato solitário localiza-se no tronco encefálico e participa do processamento da informação visceral, recebendo as principais vias aferentes do nervo vago [8]. Inspirações profundas promovem aumento acentuado da pressão arterial e do retorno venoso. Assim, os barorreceptores arteriais e cardiopulmonares são ativados por meio das vias aferentes vagais e glossofaríngeas, estimulando o núcleo do trato solitário e ativando mecanismos de relaxamento [8].

Dentro deste contexto, a mASR pode ser interessante em gestantes. A respiração lenta e profunda é utilizada na obstetrícia durante o preparo e no momento do parto, a fim de minimizar todo o estresse gerado nesta etapa [9]. Sabe-se que durante a gestação há redução no volume respiratório, e que exercícios capazes de aumentar o volume de ar inspirado e expirado podem ser benéficos nessa situação [10]. Alguns estudos avaliaram a VFC de gestantes e observaram aumento da modulação simpática $[1-3,11]$, entretanto até o momento não foram encontrados estudos que avaliassem a modulação autonômica de gestantes após um padrão respiratório lento e profundo.

Assim, o objetivo deste estudo foi comparar a resposta da modulação autonômica cardíaca de gestantes e não gestantes antes e após a mASR. A hipótese é de que após a realização da manobra haja uma maior modulação parassimpática, o que recomendaria a sua utilização durante o período gestacional e o preparo para o parto, uma vez que a modulação vagal está relacionada à sensação de bem estar e relaxamento.

\section{MÉTODOS}

\section{Delineamento e amostra}

Foi realizado um ensaio clínico não randomizado, com amostra de 24 mulheres, divididas igualmente em dois grupos: grupo gestante (GG) e grupo controle (GC) (não gestantes). Foram incluídas no estudo mulheres acima de 18 anos, que não fizessem uso de medicamentos betabloqueadores, não apresentassem diagnóstico de diabetes mellitus e concordassem em participar. O recrutamento das voluntárias deu-se por meio de divulgação por panfletos, internet e rádio. Para o GG foram consideradas mulheres grávidas independentemente da idade gestacional, e para o GC mulheres não grávidas. Foram excluídas do estudo as mulheres que apresentavam anemia, obesidade, problemas no desenvolvimento fetal e quando houve interferência do sinal coletado.

As mulheres selecionadas foram informadas quanto aos procedimentos e possíveis riscos e as que concordaram em participar assinaram o Termo de Consentimento Livre e Esclarecido. O estudo seguiu as normas do Comitê de Ética em Pesquisa em Seres Humanos da Universidade Federal de São Carlos, sendo aprovado conforme o protocolo de número 756.235 .

\section{Procedimentos}

Em um primeiro momento foi feita a anamnese e coletados os dados referentes à idade, peso, estatura, índice de massa corporal (IMC), pressão arterial (PA) e frequência respiratória (FR) em repouso. As voluntárias incluídas no estudo foram submetidas à avaliação da VFC antes e após uma sessão da mASR. 
Para a mASR as participantes foram posicionadas em decúbito lateral esquerdo, devido às circunstâncias anatômicas uterinas e cardíacas, sendo esta a melhor posição para evitar a compressão de grandes vasos, além de minimizar os aspectos gravitacionais que atuam sobre o ventre da gestante, possibilitando maior conforto na realização do teste [11]. A FC foi coletada por um cardiofrequencímetro (Polar, modelo S810i, Kempele, Finlândia). A cinta que fornece a informação para o cardiofrequencímetro foi colocada em contato com o tórax da voluntária por meio de uma faixa elástica, e a VFC foi mensurada durante todo o tempo da manobra.

Foi utilizado esfigmomanômetro do tipo aneróide devidamente calibrado para aferição da PA. A FR foi medida pelo método visual, contando o número de ciclos respiratórios realizados em um minuto durante o repouso pré e pós-mASR. Os intervalos RR (iRR) foram registrados de forma contínua, e durante o repouso pré e pós-mASR as voluntárias foram orientadas a respirar espontaneamente.

\section{Protocolo do controle da respiração}

Foi aplicado um protocolo de treinamento de controle respiratório, utilizando inicialmente a prática do padrão diafragmático (inspiração nasal lenta e profunda com deslocamento anterior da região abdominal) para conscientização do movimento com apoio abdominal. As voluntárias foram orientadas a observar o movimento abdominal durante a respiração. Foram solicitados 10 movimentos respiratórios para aprendizagem desse padrão antes da coleta de dados, acompanhados por comando e auxílio verbal. Após esse treinamento as voluntárias foram orientadas a permanecer em repouso por mais 10 minutos antes do início da coleta, para que o procedimento de familiarização não influenciasse nos dados da VFC pré-mASR.

A coleta da VFC foi realizada com duração total de 12 minutos. Nos primeiros quatro minutos, as participantes foram orientadas a respirar espontaneamente para que fossem mensurados os dados em estado de repouso. Do quinto ao oitavo minuto, as voluntárias realizaram a mASR, controlada a uma taxa de seis ciclos respiratórios por minuto, sendo cinco segundos para inspiração e cinco segundos para expiração. No momento de execução dessa manobra a avaliadora forneceu comando e incentivo verbais, tanto no período inspiratório quanto no expiratório, orientando que a voluntária "puxe" e "solte" o ar. Ao término do oitavo minuto, a PA foi mensurada e as participantes voltaram a respirar dentro do volume corrente espontâneo. Durante toda a respiração espontânea o ambiente permaneceu silencioso e a voluntária imóvel, para que ruídos ou movimentos bruscos não prejudicassem a coleta dos dados referentes ao repouso.

\section{Processamento dos dados}

A seleção do trecho do registro para análise das condições de repouso foi realizada por meio da inspeção visual da distribuição dos iRR (milissegundos) no período de quatro minutos, onde foi selecionado o período com maior estabilidade do sinal e que apresentasse 256 iRR, conforme preconizado pelo Task Force de 1996 [12]. Para a análise linear de repouso pré e pós-manobra, a VFC foi analisada nos domínios do tempo e da frequência por meio da transformação rápida de Fourier a partir de uma rotina específica desenvolvida no aplicativo Kubios HRV versão 2.0.

O domínio do tempo foi utilizado por expressar os dados em unidades de tempo (milissegundos), medindo-se cada intervalo RR durante o intervalo de tempo e, a partir daí, calcular os índices tradutores da variabilidade da frequência cardíaca com base em métodos estatísticos e geométricos (média, desvio padrão e índices derivados do histograma) [5]. Para a análise no domínio do tempo foi utilizado o índice "raiz quadrada da média das diferenças sucessivas ao quadrado entre os iRR adjacentes dividido pelo número de iRR menos um" (RMSSD), expresso em milissegundos $[5,12]$.

O domínio da frequência, método de análise mais utilizado atualmente, consiste em decompor a VFC em componentes oscilatórios fundamentais [5]. Para a análise do domínio da frequência foram consideradas as duas bandas de frequência que melhor representam a atuação dos componentes simpático e vagal sobre o controle da FC: a banda de baixa frequência (BF), atribuída predominantemente à modulação simpática; $\mathrm{e}$ a banda de alta frequência (AF), atribuída à modulação vagal. Esses componentes foram determinados em valores normalizados (un) segundo as padronizações descritas no Task Force de 1996: "obtido pela divisão do componente espectral em questão pela densidade espectral total, subtraída do componente de muito baixa frequência e multiplicada por 100". Adicionalmente, o balanço simpato-vagal foi expresso pela razão BF/AF [12].

Além dos métodos lineares, foram utilizados métodos não lineares de análise da VFC para detectar alterações que podem não ser reconhecidas pelos 
métodos tradicionais. Diversos estudos mostram que os sistemas não lineares têm sido progressivamente aplicados para esclarecer o comportamento dos fenômenos biológicos. Esses parâmetros têm se mostrado bons preditores de morbimortalidade no âmbito clínico e podem ser obtidos pela análise da complexidade de sinais biológicos e pela análise de Poincaré, por exemplo $[5,13,14]$. A entropia de Shanon foi utilizada como medida de complexidade e obtida através de um histograma com a frequência de aparecimento dos iRR. Além disso, a análise de Poincaré foi feita a partir dos índices SD1 e SD2, que são utilizados para identificar a VFC global, utilizando o registro instantâneo da variabilidade batimento a batimento e a razão entre as variações curta e longa dos iRR [5, 14].

Para a análise dos ciclos respiratórios da mASR foram calculados os índices de acordo com a padronização descrita pelo Task Force (1996): razão expiração/inspiração (E/I) - média dos maiores valores de iRR obtidos durante a fase expiratória dividido pelas médias dos menores valores $i R R$ da fase inspiratória; e a diferença inspiração-expiração $(\Delta \mathrm{IE})$ diferença entre a média dos maiores valores de FC obtidos durante a fase inspiratória e a média dos menores valores de FC durante a fase expiratória da mASR [12].

\section{Análise estatística}

Foi realizado um cálculo amostral pelo do programa G*Power 3.1.3, baseado na variável de desfecho que representa o balanço simpato-vagal (relação BF/AF), e observou-se que, para se atingir um poder de $80 \%$, com tamanho de efeito de 0,25 e nível de significância de $5 \%$, seriam necessários 22 indivíduos, divididos igualmente entre os grupos.

Inicialmente foi empregado o teste KolmogorovSmirnov para verificar a normalidade dos dados obtidos. Para comparar as características dos sujeitos (peso, altura, idade, IMC), bem como os índices da VFC e da mASR intergrupo, foi aplicado o teste $t$ de Student. Para a comparação intragrupo foi utilizado o teste t de Student pareado. Os índices da mASR (E/I e $\Delta \mathrm{IE})$ não apresentaram distribuição normal e foram transformados em valores logarítmicos decimais para o tratamento estatístico. As análises foram consideradas com nível de significância estabelecido de $5 \%$.

\section{RESULTADOS}

As características gerais das mulheres avaliadas estudo estão apresentadas na Tabela 1. A média de idade das participantes foi de $28 \pm 3$ anos para o GG e $28 \pm 5$ anos para o GC. A média da idade gestacional foi de $23,2 \pm 7,8$ semanas. Não houve diferença significativa entre os grupos para as variáveis: idade, peso antes da gestação, estatura e IMC pré-gestacional.

Tabela 1. Caracterização da amostra de gestantes (GG) e mulheres não gestantes (GC) incluídas no estudo.

\begin{tabular}{lccc}
\hline Variável & $\begin{array}{c}\text { GG } \\
(\mathbf{n = 1 2})\end{array}$ & $\begin{array}{c}\text { GC } \\
(\mathbf{n = 1 2})\end{array}$ & p valor \\
\hline Idade (anos) & $28 \pm 3$ & $28 \pm 5$ & 0.371 \\
Peso Antes da Gestação $(\mathrm{kg})$ & $58,4 \pm 7,2$ & $60,0 \pm 6,9$ & 0.282 \\
Estatura (cm) & $171,2 \pm 5,1$ & $168,5 \pm 3,7$ & 0.671 \\
IMC antes da gestação $\left(\mathrm{kg} / \mathrm{cm}^{2}\right)$ & $22,9 \pm 1,5$ & $21,3 \pm 2,1$ & 0.228 \\
IG (semanas) & $23,2 \pm 7,8$ & - & \\
Peso Adquirido na Gestação $(\mathrm{kg})$ & $7,4 \pm 6,9$ & - & \\
\hline
\end{tabular}

Dados expressos em média e desvio padrão.

GG, grupo gestante; GC, grupo controle; IMC, índice de massa corporal; IG, idade gestacional.

Os dados representativos das variáveis cardiovasculares lineares e não lineares estão apresentados na Tabela 2. Na comparação entre os grupos no momento pré-mASR podemos observar os índices FC, BF e $\mathrm{BF} / \mathrm{AF}$ mais elevados e $\mathrm{AF}$ diminuída no grupo $\mathrm{GG}$ em relação ao GC. Já na comparação entre os grupos pós-mASR, o GG apresentou maior média da FC e maior índice RMSSD que o GC, o que mostra aumento da modulação vagal nas gestantes após a manobra respiratória. Ainda, o GG apresentou valores mais elevados de BF, BF/AF e entropia de Shannon após a mASR, em comparação ao GC.

$\mathrm{Na}$ comparação entre pré e pós-mASR, para o GC foram observados maiores valores da entropia de Shanon no momento pós-manobra. Já na comparação pré e pós-mASR para o GG, foram observados maiores valores e de SD1 no momento pré-manobra e maiores valores de RMSSD (indicando aumento da modulação vagal) e entropia de Shannon no momento pós-manobra.

As razões $\mathrm{E} / \mathrm{I}$ e o $\Delta \mathrm{IE}$ durante a realização da mASR foram maiores no $\mathrm{GC}$ e estão representadas na Tabela 3. 
Tabela 2. Comparação de variáveis cardiovasculares entre os grupos pré e pós-manobra de acentuação da arritmia sinusal respiratória, em gestantes (GG) e mulheres não gestantes (GC).

\begin{tabular}{|c|c|c|c|c|c|c|c|c|}
\hline & \multicolumn{2}{|c|}{ GG $(n=12)$} & \multicolumn{2}{|c|}{ GC $(n=12)$} & \multicolumn{2}{|c|}{ p valor - intragrupo } & \multicolumn{2}{|c|}{ p valor - intergrupo } \\
\hline & Pré-mASR & Pós-mASR & Pré-mASR & Pós-mASR & Pré-mASR & Pós-mASR & GG & GC \\
\hline \multicolumn{9}{|l|}{ Índices lineares } \\
\hline iRR Média (ms) & $722,7 \pm 74,1$ & $726,1 \pm 47,4$ & $829,7 \pm 87,6+$ & $853,1 \pm 67,2^{*}$ & 0,032 & 0,031 & 0,532 & 0,621 \\
\hline $\mathrm{BF}$ (un) & $68,9 \pm 28,1$ & $65,2 \pm 14,4$ & $49,3 \pm 11,7^{+}$ & $47,3 \pm 17,8^{*}$ & 0,002 & 0,020 & 0,143 & 0,251 \\
\hline $\mathrm{AF}$ (un) & $31,1 \pm 28,1$ & $34,8 \pm 14,4$ & $50,7 \pm 11,7^{+}$ & $52,7 \pm 20,1^{*}$ & 0,011 & 0,041 & 0,215 & 0,092 \\
\hline $\mathrm{BF} / \mathrm{AF}$ & $7,1 \pm 8,4$ & $2,6 \pm 2,0$ & $1,1 \pm 0,6^{+}$ & $1,0 \pm 0,9 *$ & 0,025 & 0,001 & 0,076 & 0,124 \\
\hline RMSSD (ms) & $34,5 \pm 5,7$ & $38,9 \pm 5,8^{\ddagger}$ & $25,9 \pm 2,6$ & $27,1 \pm 3,9^{*}$ & 0,154 & 0,025 & 0,027 & 0,097 \\
\hline FC Média (bpm) & $87,4 \pm 10,1$ & $85,3 \pm 6,0$ & $72,4 \pm 9,5^{+}$ & $69,7 \pm 3,5^{*}$ & 0,021 & 0,007 & 0,132 & 0,087 \\
\hline \multicolumn{9}{|l|}{ Índices não lineares } \\
\hline SD1 (ms) & $23,7 \pm 18,6$ & $13,7 \pm 4,3^{\ddagger}$ & $19,8 \pm 1,9$ & $18,9 \pm 2,8^{*}$ & 0,231 & 0,043 & 0,031 & 0,166 \\
\hline $\mathrm{SD} 2(\mathrm{~ms})$ & $68,1 \pm 39,0$ & $49,2 \pm 9,7$ & $49,8 \pm 12,2$ & $50,8 \pm 7,9$ & 0,163 & 0,098 & 0,276 & 0,216 \\
\hline Shannon & $2,9 \pm 0,2$ & $3,1 \pm 0,2^{\ddagger}$ & $2,8 \pm 0,2$ & $3,0 \pm 0,2 * \S$ & 0,251 & 0,032 & 0,002 & 0,003 \\
\hline
\end{tabular}

Dados expressos em média e desvio padrão.

GG, grupo gestante; GC, grupo controle; mASR, manobra de acentuação da arritmia sinusal respiratória; BF, banda de baixa frequência; AF, banda de alta frequência; RMSSD, raiz quadrada da média do quadrado das diferenças entre os intervalos RR normais adjacentes; FC, frequência cardíaca; SD1, variabilidade batimento a batimento; SD2, desvio padrão a longo prazo da variabilidade.

* diferença entre os grupos pós-mASR; † diferença entre os grupos pré-mASR; ‡ diferença entre pré e pós-mASR para o GG; § diferença entre pré e pós-mASR para o GC.

Tabela 3. Comparação entre os índices da variabilidade da frequência cardíaca obtidos durante a manobra de acentuação da arritmia sinusal respiratória, entre gestantes (GG) e mulheres não gestantes (GC).

\begin{tabular}{cccc}
\hline Variável & GG & GC & p valor \\
$\mathrm{E} / \mathrm{I}$ & $1,17 \pm 0,6$ & $1,20 \pm 0,3$ & $\mathbf{0 , 0 1 7}$ \\
$\Delta \mathrm{IE}$ & $12,1 \pm 6,2$ & $13,5 \pm 2,8$ & 0,021 \\
\hline
\end{tabular}

Dados expressos em média e desvio padrão.

GG, grupo gestante; GC, grupo controle; mASR, manobra de arritmia sinusal respiratória; $\mathrm{E} / \mathrm{I}$, razão expiração/inspiração; $\Delta \mathrm{IE}$, diferença entre a média dos maiores valores de FC obtidos durante a fase inspiratória e a média dos menores valores de FC durante a fase expiratória.

\section{DISCUSSÃO}

Os principais achados do presente estudo demonstram que: i) as gestantes apresentaram média da FC de repouso mais elevada que as não gestantes; ii) a análise linear no domínio do tempo e da frequência mostrou maiores valores basais de $\mathrm{BF}$ e menores valores de AF e RMSSD para GG quando comparados ao GC; iii) a análise linear não foi capaz de detectar diferenças entre os momentos pré e pós para o GC e mostrou aumento apenas do índice RMSSD na comparação pré-manobra vs pós-manobra no GG, o que indica aumento da modulação vagal nas gestantes após a manobra respiratória; iv) a partir da análise dos índices não lineares foi possível observar aumento da complexidade após a mARS para ambos os grupos, embora esse índice tenha sido mais elevado no GG.

Em relação aos dados antropométricos e clínicos, nenhum dos índices avaliados apresentou diferença significativa entre os grupos, o que caracteriza uma amostra homogênea. Entretanto, os maiores valores de FC e o predomínio da modulação simpática de repouso observados no GG corroboram com os achados de diversos estudos $[1-3,15,16]$. Tejera et al. [1] avaliaram 563 mulheres e, assim como no presente estudo, observaram maiores valores de FC e atuação simpática no grupo de gestantes. Estes achados podem ser explicados, uma vez que o sistema nervoso autônomo atua constantemente para modular o funcionamento de vários sistemas orgânicos [5]. Assim, este sistema é o principal responsável pelo aumento da frequência cardíaca, que é mais elevada em mulheres no período gestacional a fim de suprir as alterações anatômicas e fisiológicas e garantir adequado aporte sanguíneo para o feto [1-3, 16-18].

Embora a análise linear no domínio da frequência não tenha apontado diferença estatística nos momentos pré $v s$ pós-mASR, podemos observar aumento dos valores de $\mathrm{AF}$ em ambos os grupos, sendo maior no GG. Além disso, a análise do domínio do tempo mostrou valores significativamente mais elevados para o índice RMSSD após a mASR nas gestantes, o que indica maior modulação vagal após a manobra. Este aumento da modulação parassimpática concorda com a hipótese do estudo e pode ser um indicativo clínico de relaxamento, uma vez que a manobra estimula o aumento do volume corrente, o que diminui a modulação simpática e produz sensação de bemestar e redução da dor [8, 19-22]. Miyazato et al. [19] mostraram que a redução de índices da modulação parassimpática estão relacionadas à sensação de 
relaxamento materno e podem promover aumento da dilatação cervical durante o trabalho de parto. Assim, a mASR poderia ser indicada durante todo o processo gestacional e no período do parto com esta finalidade.

A análise não linear evidenciou aumento significativo das medidas de complexidade (entropia de Shanon) após mASR em ambos os grupos, sendo maior nas gestantes. De acordo com Moertl et al. [23], que acompanharam a modulação autonômica de 36 mulheres durante o curso da gravidez, os sistemas individuais tornam-se mais independentes entre si e diminuem a troca de informações ao tentar atender às demandas cardiovascular e respiratórias maternas. $\mathrm{O}$ aumento da complexidade indica que uma maior integração entre os sistemas fisiológicos pôde ser observada ao final da manobra, sugerindo melhora na troca de informações entre os sistemas [14, 24], como, por exemplo, os centros respiratórios, de pressão e de controle da dor.

De acordo com Souza e Ferreira [25], para o funcionamento vagal ser considerado íntegro, a razão E/I também deve ser analisada e apresentar valores positivos maiores que um. Ainda, May et al. [16] mostraram que exercícios associados à respiração promoveram melhora do controle autonômico em mulheres grávidas. No presente estudo, observamos valores indicativos da integridade do sistema nervoso autônomo avaliado pela mASR para ambos os grupos, embora tais respostas sejam atenuadas nas gestantes, indicando uma capacidade de ajuste rápido do sistema nervoso autônomo mais eficiente no grupo de não gestantes.

Apesar dos indicativos clínicos de redução da ativação simpática e aumento da modulação vagal após a mASR observados no presente estudo, sugerimos que os iRR sejam coletados em um tempo maior de avaliação, para que os efeitos possam ser testados a longo prazo com maior precisão. Além disso, como limitação do presente estudo, destaca-se que foram incluídas gestantes em diferentes idades gestacionais. Acreditamos que, devido a todas as diferenças anatômicas e fisiológicas ao longo do período gestacional, é importante explorar a análise da VFC e da mASR nos diferentes trimestres da gestação.

Foi confirmada a hipótese de aumento da modulação vagal nas gestantes após a mASR, o que sugere efeito de relaxamento e aumento da sensação de bem-estar para essa população e pode ser importante durante todo o período gestacional. Além disso, observou-se aumento dos índices de complexidade em ambos os grupos após a manobra, sugerindo aumento da integração dos sistemas fisiológicos e, além disso, produzindo efeito cardioprotetor. Tais achados podem consubstanciar a importância da manobra de respiração lenta e profunda no período gestacional. No entanto, futuros estudos são necessários para avaliar os efeitos desta manobra durante o parto.

\section{NOTAS}

Agradecimentos

Os autores agradecem à Fundação de Amparo à Pesquisa do Estado de São Paulo pelo suporte financeiro destinado ao desenvolvimento deste estudo.

\section{Apoio financeiro}

Fundação de Amparo à Pesquisa do Estado de São Paulo (FAPESP) - Processo de número 2012/21909-4.

\section{Declaração de conflito de interesses}

Os autores declaram não haver conflitos de interesses relevantes ao conteúdo deste estudo, informam ter tido acesso a todos os dados obtidos e assumem completa responsabilidade pela integridade dos resultados.

\section{REFERÊNCIAS}

1. Tejera E, Areias JM, Rodrigues A, Nieto-Villar JM, Rebelo I. Blood pressure and heart rate variability complexity in normal pregnancy. Influence of age, familiar history and parity. Hypertens Pregnancy. 2012;31(1):91-106. https://doi.or $\mathrm{g} / 10.3109 / 10641955.2010 .544801$

2. Tejera E, Areias JM, Rodrigues A, Rebelo I, Nieto-Villar JM. Network centrality and multiscale transition asymmetry in the heart rate variability analysis of normal and preeclamptic pregnancies. Commun Nonlinear Sci Numer Simul. 2011;16(3):1589-96. https://doi.org/10.1016/j.cnsns.2010.07.009

3. Tejera E, Areias MJ, Rodrigues AI, Ramõa A, Nieto-Villar JM, Rebelo I. Relationship between heart rate variability indexes and common biochemical markers in normal and hypertensive third trimester pregnancy. Hypertens Pregnancy. 2012b;31(1):59-69. https://doi.org/10.3109/10641955.2010.544802

4. Kamath M, Watanabe M, Upton A. Heart rate variability signal analysis: clinical applications. New York: CRC Press; 2013.

5. Vanderlei LCM, Pastre CM, Hoshi RA, Carvalho TD, Godoy MF. Basic notions of heart rate variability and its clinical applicability. Braz J Cardiovasc Surg. 2009;24(2):205-17. https://doi.org/10.1590/S0102-76382009000200018 
6. Ferreira LL, Souza NM, Bernardo AFB, Vitor ALR, Valenti VE, Vanderlei LCM. Heart rate variability as a resource in physical therapy: analysis of national journal. Fisioter Mov. 2013;26(1):25-36. https://doi.org/10.1590/S010351502013000100003

7. Hayano J, Mukai S, Sakakibara M, Okada A, Takata K, Fujinami T. Effects of respiratory interval on vagal modulation of heart rate. Am J Physiol. 1994;267(1):H33-40.

8. Bruehl S, Chung OY. Interactions between the cardiovascular and pain regulatory systems: an updated review of mechanisms and possible alterations in chronic pain. Neurosci Biobehav Rev. 2004;28(4):395-414. https://doi.org/10.1016/j. neubiorev.2004.06.004

9. Almeida L, Constâncio J, Santos C, Silva T, Raposo M. Análise comparativa das PE e PI máximas entre mulheres grávidas e não-grávidas e entre grávidas de diferentes períodos gestacionais. Rev Saúde Com. 2005;1(1):9-17.

10. Duarte G. Modificações e adaptações do organismo materno decorrentes da gravidez. In: Ferreira CHJ, Carvalho CRF, Tanaka C. Fisioterapia na saúde da mulher. Rio de Janeiro: Guanabara Koogan; 2011.

11. Heiskanen N, Saarelaine H, Valtonen P, Laitinen T, Vanninen E, Heinonen S. Blood pressure and heart rate variability analysis of orthostatic challenge in normal human pregnancies. Clinic Physiol Function Imaging. 2008;28(6):384-90. https://doi.org/10.1111/j.1475-097X.2008.00818.x

12. Task Force of the European Society of Cardiology and the North American Society of Pacing and Electrophysiology. Heart rate variability: standards of measurement, physiological interpretation and clinical use. Circulation. 1996;93(5):1043-65. https://doi.org/10.1161/01.CIR.93.5.1043

13. Logier R, De Jonckheere J, Dassonneville A. An efficient algorithm for R-R intervals series filtering. Conf Proc IEEE Eng Med Biol Soc. 2004;6:3937-40. https://doi.org/10.1109/IEMBS.2004.1404100

14. Porta A, Faes L, Masé M, D'Aggio G, Pinna GD, Maestri R, Montano N, Furlan R, Guzzetti S, Nollo G, Malliani A. An integrated approach based on uniform quantization for the evaluation of complexity of short-term heart period variability: Application to $24 \mathrm{~h}$ Holter recordings in healthy and heart failure humans. Chaos. 2007;17(1):015117. https:// doi.org/10.1063/1.2404630

15. Mizuno T, Tamakoshi K, Tanabe K. Anxiety during pregnancy and autonomic nervous system activity: A longitudinal observational and cross-sectional study. J Psychosom Res. 2017;99:105-111. https://doi.org/10.1016/j.jpsychores.2017.06.006

16. May LE, Knowlton J, Hanson J, Suminski R, Paynter C, Fang X, Gustafson KM. Effects of Exercise During Pregnancy on Maternal Heart Rate and Heart Rate Variability. PMR. 2016;8(7):611-7. https://doi.org/10.1016/j.pmrj.2015.11.006

17. Nakagaki A, Inami T, Minoura T, Baba R, Iwase S, Sato M. Differences in autonomic neural activity during exercise between the second and third trimesters of pregnancy. J Obstet Gynaecol Res. 201;42(8):951-9.

18. Carpenter RE, Emery SJ, Uzun O, Rassi D, Lewis MJ. Influence of antenatal physical exercise on heart rate variability and QT variability. J Matern Fetal Neonatal Med. 2017;30(1):79-84. https://doi.org/10.3109/14767058.2016.1163541

19. Miyazato K, Matsukawa K. Decreased cardiac parasympathetic nerve activity of pregnant women during foot baths. Jpn J Nurs Sci. 2010;7(1):65-75. https://doi.org/10.1111/j.1742-7924.2010.00136.x

20. Arsenault M, Ladouceur A, Lehmann A, Rainville P, Piche M. Pain modulation induced by respiration: phase and frequency effects. Neuroscience. 2013;252:501-11. https://doi.org/10.1016/j.neuroscience.2013.07.048

21. Yasuma F, Hayano J. Respiratory Sinus Arrhytmia: why does the heartbeat synchronize with respiratory rhythm? Chest. 2004;125(2):683-90. https://doi.org/10.1378/chest.125.2.683

22. DiPietro JA, Costigan KA, Nelson P, Gurewitsch ED, Laudenslager ML. Fetal responses to induced maternal relaxation during pregnancy. Biol Psychol. 2008;77(1):11-9. https://doi.org/10.1016/j.biopsycho.2007.08.008

23. Moertl MG, Lackner HK, Papousek I, Roessler A, Hinghofer-Szalkay H, Lang U, Kolovetsiou-Kreiner V, Schlembach D. Phase synchronization of hemodynamic variables at rest and after deep breathing measured during the course of pregnancy. PLoS One. 2013;8(4):e60675. https://doi.org/10.1371/journal.pone.0060675

24. Goldberger AL. Non-linear dynamics for clinicians: chaos theory, fractals, and complexity at the bedside. Lancet. 1996;347(9011):1312-4. https://doi.org/10.1016/S0140-6736(96)90948-4

25. Souza AI, Filho MB, Ferreira LOC. Alterações hematológicas e gravidez. Revista Brasileira de Hematologia e Hemoterapia. 2002;24(1):29-36. https://doi.org/10.1590/S1516-84842002000100006 